\title{
Indicadores nutricionais combinados e fatores associados em população Quilombola no Sudoeste da Bahia, Brasil
}

\author{
Combined nutritional indicators and associated factors \\ in the Quilombo population (hinterland settlements \\ founded by people of African origin) in southwestern Bahia, Brazil
}

Daniela Arruda Soares ${ }^{1}$

Sandhi Maria Barreto ${ }^{2}$
${ }^{1}$ Núcleo de Epidemiologia e Saúde Coletiva,

Universidade Federal da Bahia, Campus

Anísio Teixeira, Instituto Multidisciplinar em Saúde.

R. Rio de Contas 58/Quadra 17/Lote 58, Candeias 45029-094. Vitória da Conquista BA Brasil. dandani23@yahoo.com.br

${ }^{2}$ Departamento de Medicina Preventiva e Social, Faculdade de Medicina, Universidade Federal de Minas Gerais.

\begin{abstract}
This study sought to estimate the prevalence of the combination of Body Mass Index (BMI) plus Waist Circumference (WC) and of BMI plus Waist to Height Ratio (WHR) and to investigate associated factors. A two-stage random sample of adults ( $>20$ years) living in former African slaves communities (Quilombos) was taken in Vitória da Conquista in the State of Bahia in 2011. Combined BMI+WHR nutritional risk was defined by the simultaneous presence of BMI > $25.0 \mathrm{Kg} / \mathrm{m} 2$ or $>27.0 \mathrm{Kg} / \mathrm{m} 2$, if aged $>60$ years and WHR $>0.5$. BMI + WC nutritional risk was defined by elevated BMI in addition to WC $>80 \mathrm{~cm}$ for women, or $>94 \mathrm{~cm}$ for men. Among the 739 participants, the prevalence of combined nutritional indicators were $35.3 \%$ (BMI + WHR) and $26.8 \%(B M I+W C)$. Female sex and hypertension increased the chances of a combination of both indicators, while being unmarried decreased the chances. The prevalence of BMI +WHR was higher in the 40-59 year age range and the prevalence of BMI + WC was higher in the 40-49 year age range. Less schooling increased the chances of the combined BMI + WHR indicator and watching television for more than two hours/day increased the chances of the BMI +WC indicator. The high prevalence of combined nutritional indicators indicate the need of diet promotion actions to prevent obesity.
\end{abstract}

Key words Overweight, Obesity, Abdominal obesity, Height, African continental ancestry group
Resumo Este estudo estimou a prevalência de duas combinações de indicadores nutricionais: Indice de Massa Corporal (IMC) + Circunferência da Cintura (CC) e IMC + Razão Cintura Estatura (RCE), e investigou os fatores associados em adultos Quilombolas. Amostra aleatória em dois estágios de 739 adultos $\geq 20$ anos, residentes em Comunidades Quilombolas em Vitória da Conquista (BA), em 2011. Risco combinado IMC + $R C E$ incluiu os indivíduos que simultaneamente apresentaram $I M C \geq 25,0 \mathrm{Kg} / \mathrm{m} 2$ ou $\geq 27,0 \mathrm{Kg} / \mathrm{m} 2$, se idade $\geq 60$ anos e $R C E \geq 0,5$. O risco IMC + RCE incluiu indivíduos com IMC elevado que também apresentavam $C C \geq 80 \mathrm{~cm}$, se mulher, ou $\geq 94 \mathrm{~cm}$, se homem. As prevalências dos indicadores nutricionais combinados foram de 35,3\% (IMC + RCE) e 26,8\% (IMC + CC). Sexo feminino e hipertensão aumentaram a chance para os dois indicadores enquanto que o estado civil não casado diminuiu esta chance. O indicador IMC + RCE foi maior na faixa etária de 40 a 59 anos e o indicador IMC + CC foi mais frequente na faixa de 40 a 49 anos. A baixa escolaridade elevou o indicador IMC $+R C E$, enquanto assistir televisão por mais de duas horas por dia, o indicador IMC + CC. A elevada prevalência de risco nutricional para DCNT, especialmente de obesidade central, confirmou a necessidade de ações de promoção de dieta saudável e saúde.

Palavras-chave Sobrepeso, Obesidade, Obesidade abdominal, Estatura, Grupo com ancestrais do continente africano 


\section{Introdução}

As doenças crônicas não transmissíveis (DCNT) constituem a principal causa de morte no Brasil e o grupo que mais contribui para a carga global de doenças ${ }^{1}$. Dentre os principais fatores de risco para as doenças crônicas, destacam-se o sobrepeso e a obesidade, com prevalências cada vez mais elevadas nos países de renda alta e média, incluindo o Brasil ${ }^{2}$. Este quadro reflete a transição nutricional em curso, e ocorre concomitante a mudanças demográficas e epidemiológicas aceleradas $^{3}$. Esses distúrbios nutricionais são associados à elevação da morbimortalidade por DCNT em estudos epidemiológicos longitudinais e transversais ${ }^{4-7}$, contudo, pouco se sabe sobre sua ocorrência entre minorias populacionais como a população Quilombola.

As comunidades quilombolas, reconhecidas apenas recentemente pela Constituição Brasileira, são autodefinidas a partir das relações com a terra, parentesco, práticas culturais e presunção de ancestralidade negra ${ }^{8}$. Elas representam o resgate de uma dívida histórica com a população afro-descendente, a qual constituiu os Quilombos em sua luta contra a opressão sofrida e em prol da liberdade do regime escravocrata que vigeu no país no período colonial ${ }^{9}$.

Estima-se que existam cerca de 1,17 milhões de quilombolas e 1948 comunidades oficialmente reconhecidas no Brasil, sendo que a região Nordeste registra o maior contingente ${ }^{10}$. Estudos mostram indícios de transformações socioeconômicas e culturais nessas comunidades, incluindo costumes, hábitos de saúde e alimentares, resultantes do maior contato destes povos com a sociedade em geral ${ }^{11,12}$. Além disso, dificuldades de titulação fundiária, escassez de terras para plantio, piora das secas no Nordeste, e o maior contato com alimentos industrializados também concorrem para mudanças no modo de vida e padrões nutricionais nessas comunidades ${ }^{12,13} \mathrm{e}$ entre outras populações tradicionais ${ }^{14-16}$.

A avaliação do estado nutricional geralmente se baseia no índice de massa corporal (IMC), apesar desta medida não discriminar o padrão de distribuição da gordura e sim a proporção corporal $^{17,18}$. Medidas que avaliam a concentração de gordura na região abdominal têm apresentado maior sensibilidade preditiva para as DCNT ${ }^{5,6,19}$. A circunferência da cintura (CC) e a razão cintura-estatura (RCE) são exemplos de medidas que avaliam a concentração de gordura central/ abdominal. A CC não considera as diferenças de estatura entre os indivíduos ${ }^{20}$, por isto, a razão cintura-estatura vem sendo apontada como um bom preditor de risco cardiovascular ${ }^{6,21,22}$, além de minimizar as diferenças devido à estatura $\mathrm{e}$ sexo e poder ser utilizado em diferentes etnias. A combinação de diferentes indicadores para avaliar gordura corporal e central, representa uma prática recente ${ }^{23-25}$ e pode concorrer para aumentar a acurácia do diagnóstico nutricional ${ }^{17}$.

O objetivo deste estudo foi estimar a prevalência de indicadores nutricionais combinados [índice de massa corporal (IMC) e razão cintura estatura (RCE), e índice de massa corporal (IMC) e circunferência da cintura (CC)] e analisar associações entre fatores socioeconômicos, demográficos, comportamentais e de saúde com os dois tipos de indicadores nutricionais combinados, entre adultos residentes em Comunidades Quilombolas na cidade de Vitória da Conquista, Bahia, Brasil.

\section{Materiais e métodos}

Entre setembro a outubro de 2011 foi realizado estudo transversal de base populacional, com indivíduos $\geq 18$ anos, denominado "Projeto Comquista: Comunidades Quilombolas de Vitória da Conquista- avaliação de saúde e seus condicionantes", no qual múltiplos desfechos de saúde foram investigados. Uma amostra representativa da população Quilombola residente na zona rural do município de Vitoria da Conquista, Bahia, foi obtida, considerando a estimação da população -alvo em 2.935 indivíduos adultos provenientes das 10 comunidades sediadas em 05 distritos da região, conforme informações da Secretária Municipal de Saúde e Fundação Palmares.

A amostra de 884 indivíduos foi calculada utilizando uma prevalência de 50\%, dado a heterogeneidade dos eventos mensurados, precisão de $5 \%$, intervalo de confiança de $95 \%$, efeito de desenho $=2$ e perdas de $30 \%$. O plano amostral foi realizado em dois estágios: inicialmente selecionou-se aleatoriamente uma comunidade por distrito, com probabilidade proporcional ao número de habitantes da comunidade, totalizando cinco comunidades (Corta Lote, Maria Clemência, Furadinho, Lagoa de Melquíades e Boqueirão) certificadas pela Fundação Palmares e, com número mínimo de 50 famílias; em um segundo momento realizou-se a seleção domiciliar conforme distribuição proporcional de domicílios por distrito. A lista de domicílios foi obtida por comunidade, e para tanto foi utilizado um sistema de georreferenciamento e fotografia dos 
domicílios, posteriormente, foi realizado sorteio aleatório sem repetição dos mesmos. Todos os adultos dos domicílios foram entrevistados.

A coleta de dados foi feita por meio de entrevista face-a-face utilizando questionários padronizados e pré-codificados, realizada nos domicílios dos participantes por entrevistadores treinados. O questionário utilizado foi baseado na Pesquisa Nacional de Saúde (PNS), o qual passou por etapas de avaliação, pré-teste e adequações. A linguagem, sequência, coerência entre as questões bem como o tempo necessário para aplicação das entrevistas foi avaliado em pré-testes e, um estudo piloto foi realizado a fim de ajustar o questionário antes da sua aplicação no estudo principal. Os dados foram coletados em computadores portáteis (HP Pocket Rx5710, HewlettPackard Development Company, Estados Unidos), posteriormente transferidos e armazenados em um banco de dados próprio, favorecendo a identificação de possíveis inconsistências e erros de digitação. Estudo piloto foi conduzido previamente para testar toda a dinâmica da coleta dos dados e a aplicabilidade do instrumento no contexto quilombola. Maiores informações metodológicas poderão ser consultadas em literatura disponível ${ }^{26}$.

Ao final das entrevistas, foram feitas medidas antropométricas e de pressão arterial (PA) por aferidores capacitados, seguindo protocolos padronizados para redução da variabilidade intra e interobservador. $\mathrm{O}$ peso foi medido em uma balança eletrônica portátil da marca Marte (Marte, São Paulo, Brasil), modelo LC200PP, com capacidade para $200 \mathrm{~kg}$ e sensibilidade de $50 \mathrm{~g}$, com os indivíduos descalços, com o mínimo de roupas e adereços. A estatura foi aferida na posição ereta, braços estendidos ao longo do corpo, descalços, cabeça erguida, com estadiômetro portátil, marca CauMaq (CauMaq Indústria Metalúrgica Ltda., Cachoeira do Sul, Brasil), modelo Est-22, com capacidade para 300 a $2000 \mathrm{~mm}$, precisão de $0,1 \mathrm{~cm}$. A circunferência da cintura foi mensurada no ponto médio situado entre a crista ilíaca e o último arco costal, com fita métrica inelástica da marca Cardiomed ${ }^{\circledR}$, modelo T-87-Wiso, capacidade de $2 \mathrm{~m}$, precisão de $0,1 \mathrm{~cm}$.

A aferição da pressão arterial foi feita em triplicata utilizando o esfigmomanômetro digital da marca Omron (OMRON Corp, São Paulo, Brasil), modelo HEM-742(OMRON Corp., São Paulo, Brasil). A coleta das medidas antropométricas foi baseada nas técnicas preconizadas por Jelliffe $^{27}$, e a aferição da PA, de acordo com o protocolo das VI Diretrizes de Hipertensão Arterial da Sociedade Brasileira de Cardiologia ${ }^{28}$. 5\% da amostra de cada comunidade foi reentrevistada e o questionário aplicado na íntegra para fins de controle de qualidade. Foram aplicados os testes Kappa para as variáveis categóricas e o Coeficiente de Correlação Intra Classe para as variáveis quantitativas visando o cálculo de reprodutibilidade. Ambos os testes indicaram uma boa confiabilidade, com resultados variando de 0,60 a 1,00 para o teste Kappa e de 0,53 a 0,97 para o Coeficiente de Correlação Intraclasse.

O Índice de Massa Corporal (IMC) foi obtido pela divisão do peso pela estatura ao quadrado, cujos pontos de corte utilizados levaram em consideração a idade, sendo considerado: normal $\left(\mathrm{IMC}<25,0 \mathrm{Kg} / \mathrm{m}^{2}\right)$, sobrepeso (IMC $\geq 25,0 \mathrm{Kg}$ ) $\left.\mathrm{m}^{2}\right)$ para adultos ${ }^{29}$, e $\left(\mathrm{IMC}<27,0 \mathrm{Kg} / \mathrm{m}^{2}\right.$ - normal e IMC $\geq 27,0 \mathrm{Kg} / \mathrm{m}^{2}$ - sobrepeso) para idosos ${ }^{30}$. Os pontos de corte para a Circunferência da Cintura (CC) foram: mulheres $<80 \mathrm{~cm}$ (normal), $\geq 80 \mathrm{~cm}$ (aumentado) e, homens $<94 \mathrm{~cm}$ (normal), e $\geq$ $94 \mathrm{~cm}$ (aumentado) ${ }^{29}$. A razão cintura-estatura (RCE) foi obtida pela divisão da cintura pela estatura sendo adotados os pontos de corte de $<$ 0,5 (normal) e $\geq 0,5$ (aumentada) para ambos os $\operatorname{sexos}^{21,22}$.

Para a definição dos indicadores nutricionais combinados, utilizaram-se duas associações de critérios antropométricos: uma combinando o IMC e a RCE (IMC + RCE) e outra combinando o IMC e a CC (IMC + CC). No primeiro critério (IMC + CC), foram classificados como de risco, os indivíduos que preencheram simultaneamente os seguintes critérios: $\mathrm{IMC} \geq 25,0 \mathrm{Kg} / \mathrm{m}^{2}$ ou $\geq$ $27,0 \mathrm{Kg} / \mathrm{m}^{2}$, se idade $\geq 60$ anos e $\mathrm{RCE} \geq 0,5$. No segundo critério (IMC + RCE), foram classificados como de risco os indivíduos com IMC $\geq$ $25,0 \mathrm{Kg} / \mathrm{m}^{2}$ (ou $\geq 27,0 \mathrm{Kg} / \mathrm{m}^{2}$, se idade $\geq 60$ anos) e $\mathrm{CC} \geq 80 \mathrm{~cm}$, se mulher, ou $\geq 94 \mathrm{~cm}$, se homem. Os indivíduos que preencheram os critérios de risco acima foram comparados aos que não apresentavam risco no referido critério.

As variáveis independentes foram agrupadas em três grupos: características sociodemográficas, comportamentais e de saúde. As características sociodemográficas foram: sexo (masculino e feminino), faixa etária em anos completos (20-29 anos; 30-39 anos; 40-49 anos; 50-59 anos; $\geq 60$ anos), escolaridade em anos completos de estudo com aprovação ( 0 ; 1 -3 anos; $4-7$ anos; $\geq 8$ anos), cor da pele autorreferida (preta; parda; branca e outras), renda familiar per capita em reais, expressa em quatro percentis $\left(1^{\circ}=\leq 50 ; 2^{\circ}=51\right.$ a $119 ; 3^{\circ}=120$ a $268 ; 4^{\circ}=\geq 269$ ), estado civil (casado/união consensual; Solteiro/separado/ 
divorciado/viúvo) e índice de bens obtido pela soma total da posse de bens e utensílios domésticos $(\leq 3 ; 4-5 ; \geq 6)$ sendo considerado o relato dos seguintes bens: (televisão em cores, fogão à gás, geladeira, freezer, rádio,vídeo/DVD, máquina de lavar roupa, linha de telefone fixo, linha de telefone celular, computador, forno microondas, carro, motocicleta), situação ocupacional (trabalha; não trabalha).

As variáveis comportamentais foram: tabagismo (sim e não), tempo assistindo televisão fora do trabalho $(<2 \mathrm{~h} /$ dia, $\geq 2 \mathrm{~h} /$ dia $)$; nível de atividade física (não realiza ou faz $<3$ dias por semana; $\geq 3$ dias por semana $\geq 30 \mathrm{~min}$ ), frequência semanal de consumo de frutas e frequência semanal de consumo de verduras/legumes categorizadas em $<5$ dias/semana $\mathrm{e} \geq 5$ dias/semana e o comportamento ao comer frango com pele/ carne vermelha com gordura dicotomizada em sim e não. As características de saúde foram: autoavaliação de saúde (muito boa e boa; regular; e ruim e muito ruim), diabetes autorreferida com respostas dicotômicas (sim e não); e a hipertensão arterial sistêmica definida como pressão arterial $\geq 140$ x $90 \mathrm{mmHg}$, obtida por meio da média das duas últimas mensurações de pressão arterial nos indivíduos ou o uso habitual de medicação anti-hipertensiva. Características sociodemográficas, comportamentais e de saúde, foram autorreferidas, exceto a pressão arterial.

Foram abordados 422 domicílios, totalizando 943 indivíduos. Desses indivíduos, 15,5\% (n =146) não participaram pelos seguintes motivos: $12,7 \%(\mathrm{n}=120)$ não foram encontrados após três visitas e 2,8\% $(n=26)$ recusaram. A perda foi maior entre aqueles do sexo masculino $(77,4 \%$, $\mathrm{n}=89)$ e a faixa etária de 18 a 34 anos $(65,2 \%$, $\mathrm{n}=75)$.

Mulheres grávidas $(1,3 \%, \mathrm{n}=11)$ e os adolescentes de 10 a 19 anos $(5,9 \%, n=47)$ foram excluídos da presente análise, restando 739 participantes. Mulheres grávidas foram excluídas do estudo, devido à interferência dessa condição nos parâmetros antropométricos.

$\mathrm{Na}$ primeira etapa da análise foi estimado o perfil nutricional, a partir das classificações do IMC, CC e RCE, de acordo com o sexo. As distribuições e freqüências dos indicadores nutricionais combinados (IMC + RCE) e (IMC + CC) e as variáveis independentes investigadas foram avaliadas por meio de prevalências, intervalos de confiança e teste do qui-quadrado e comparadas aos valores de referência para cada indicador ao nível de significância de 5\%. Regressão logística uni e multivariável e o teste de Hosmer \& Le- meshow foram utilizados para construir e verificar o ajuste dos modelos de fatores associados de forma independente aos indicadores nutricionais combinados (IMC + RCE) e (IMC + CC). O nível de significância estatística de $20 \%$ foi utilizado para a seleção de variáveis candidatas aos modelos multivariados e de $5 \%$ para o ajuste. $\mathrm{O}$ modelo multivariado final foi definido manualmente, considerando o efeito da entrada de cada variável sobre as demais variáveis já retidas no modelo.

As análises estatísticas foram realizadas no pacote estatístico Stata 10.0. Avaliando-se o efeito de agrupamento intradomiciliar decorrente da entrevista que foi realizada com todos os indivíduos do domicílio $\geq 20$ anos, não foi evidenciado efeito de cluster, optando-se, portanto, em apresentar os modelos que não consideraram o efeito de agrupamento.

A pesquisa atende todos os critérios éticos sendo que os entrevistados assinaram o Termo de Consentimento Livre e Esclarecido. A pesquisa foi aprovada pelos Comitês de Ética em Pesquisa da Faculdade São Francisco de Barreiras e pela Universidade Federal de Minas Gerais.

\section{Resultados}

Entre os 739 adultos elegíveis para o presente estudo, foram excluídos da análise alguns outliers devido a erros na mensuração de peso $(2,1 \%$, $\mathrm{n}=16)$, estatura $(3,6 \%, \mathrm{n}=27)$ e circunferência da cintura $(3,9 \%, n=29)$. Considerando os indicadores nutricionais combinados, a prevalência total segundo o critério IMC + RCE $(\mathrm{P}=$ $35,36 \%$, IC95\%: 31,82-38,89) foi 1,3 vezes maior que a prevalência de risco pelo critério IMC + CC ( $P=26,81 \%$, IC95\%:23,53-30,08). Ambos os indicadores nutricionais combinados (IMC + RCE) e (IMC + CC) apresentaram prevalências maiores entre as mulheres. Para o indicador IMC $+\mathrm{RCE}$, a prevalência foi quase duas vezes maior entre as mulheres ( $P=46,05 \%$, IC95\%: 41,02$51,07)$, quando comparada com os homens ( $\mathrm{P}=$ 22,94\%, IC95\%:18,36-27,50), enquanto que para o indicador (IMC + CC) esta diferença chegou a ser quase cinco vezes maior entre as mulheres ( $P=42,59$, IC95\%: 37,59-47,59) em relação aos homens ( $P=8,56$, IC95\%: 5,52-11,60).

A prevalência de indivíduos com adiposidade central (CC aumentada e muito aumentada e RCE aumentada) nas faixas de IMC normal, sobrepeso e obesidade foi maior entre as mulheres do que entre os homens na mesma classe de peso. 
Situação inversa foi encontrada em relação a CC aumentada na categoria de obesidade corporal, onde os homens registraram maiores prevalências. Já a categoria de RCE aumentada contabilizou a mesma totalidade dos indivíduos de ambos os sexos para a obesidade corporal. (Tabela 1).

Dentre as características sociodemográficas, mantiveram-se estatisticamente associadas aos dois indicadores de risco nutricional combinado $(\mathrm{IMC}+\mathrm{RCE})$ e $(\mathrm{IMC}+\mathrm{CC})$, o sexo feminino, a faixa etária de 50 a 59 anos para o risco (IMC + RCE) e a faixa de 40-49 anos para o risco (IMC + CC), o estado civil casado/união consensual, e entre os que não trabalham. A menor escolaridade esteve associada apenas com o risco nutricional combinado (IMC + RCE) (Tabela 2). Entre os aspectos comportamentais e de saúde, o tabagismo, comer carne com gordura, autoavaliação de saúde regular e a presença de hipertensão arterial foram associados positivamente aos dois indicadores. Passar $\geq 2$ horas por dia assistindo televisão esteve associado apenas ao indicador de risco nutricional combinado (IMC + CC) (Tabela 3).

$\mathrm{Na}$ Tabela 4 encontram-se as análises uni e multivariável para os dois indicadores de risco nutricional combinado (IMC + RCE) e (IMC + CC). A chance de risco nutricional combinado (IMC + RCE) foi quase três vezes maior entre as mulheres e maior na faixa etária de 40 a 59 anos, declinando a partir daí. Foi identificado um gradiente de aumento na chance de risco nutricional (IMC + RCE) com o aumento da escolaridade, embora o OR para a faixa de escolaridade $\geq 8$ anos de estudo tenha perdido significância estatística. Indivíduos sem companheiro apresentaram menor chance de risco nutricional combinado (IMC + RCE), enquanto ser hipertenso aumentou esta chance em 1,57 vezes. No que diz respeito ao risco nutricional combinado (IMC + CC), indivíduos do sexo feminino, com idade de 40 a 49 anos, com 4 a 7 anos de escolaridade, que assistiam televisão $\geq 2 \mathrm{~h} /$ dia e os hipertensos, apresentaram aumento na chance para este tipo de risco, enquanto a menor ocorrência deste risco ocorreu entre os indivíduos que não tem companheiro. Os ajustes dos modelos pelo teste de Hosmer \& Lemeshow foram adequados, com valores de $\mathrm{p}=0,17$ para o risco combinado IMC + RCE, e $\mathrm{p}=0,37$ para o risco combinado IMC + CC.

\section{Discussão}

O perfil nutricional de adultos residentes em comunidades quilombolas deste estudo foi caracterizado por elevadas prevalências dos dois indicadores nutricionais combinados aferidos, sendo particularmente alarmantes as prevalências encontradas entre as mulheres. Estes resultados ratificam que a obesidade desponta como um problema crônico e grave de saúde ${ }^{31,32}$ inclusive em populações socialmente vulneráveis como os quilombolas do presente estudo ${ }^{14,33}$.

Pesquisas de âmbito nacional são escassas na investigação de indivíduos que apresentem obesidade abdominal e excesso de peso simulta-

Tabela 1. Distribuição de homens e mulheres adultos residentes em Comunidades Quilombolas de acordo com a categoria de Índice de Massa Corporal (IMC), Circunferência da Cintura (CC) e Razão Cintura Estatura (RCE). Projeto COMQUISTA, 2011.

\begin{tabular}{|c|c|c|c|c|c|}
\hline \multirow[b]{2}{*}{$\begin{array}{c}\text { Categorias } \\
\text { de IMC }\end{array}$} & \multicolumn{2}{|c|}{ Categorias de $\mathrm{RCE}^{* *}$} & \multicolumn{3}{|c|}{ Categorias de $\mathrm{RCE}^{* *}$} \\
\hline & $\begin{array}{c}\text { Normal } \\
\%\end{array}$ & $\begin{array}{c}\text { Aumentado } \\
\%\end{array}$ & $\begin{array}{c}\text { Normal } \\
\%\end{array}$ & $\begin{array}{c}\text { Aumentada } \\
\%\end{array}$ & $\begin{array}{c}\text { Muito aumentada } \\
\%\end{array}$ \\
\hline Feminino & $\mathrm{n}=132$ & $\mathrm{n}=249$ & $\mathrm{n}=186$ & $\mathrm{n}=146$ & $\mathrm{n}=57$ \\
\hline Normal & 62,11 & 37,89 & 80,93 & 16,49 & 2,58 \\
\hline Sobrepeso & 10,57 & 89,43 & 21,60 & 69,60 & 8,80 \\
\hline Obesidade & 0 & 100,00 & 0 & 40,00 & 60,00 \\
\hline Masculino & $\mathrm{n}=182$ & $\mathrm{n}=146$ & $\mathrm{n}=295$ & $\mathrm{n}=24$ & $\mathrm{n}=14$ \\
\hline Normal & 70,54 & 29,46 & 96,31 & 2,05 & 1,64 \\
\hline Sobrepeso & 14,29 & 85,71 & 74,36 & 19,23 & 6,41 \\
\hline Obesidade & 0 & 100,00 & 0 & 44,44 & 55,56 \\
\hline
\end{tabular}

* IMC: Normal $\left(18,5 \geq \mathrm{IMC}<25,0 \mathrm{Kg} / \mathrm{m}^{2}\right)$, sobrepeso $\left(25,0 \geq \mathrm{IMC}<30,0 \mathrm{Kg} / \mathrm{m}^{2}\right)$ e obesidade $\left(\mathrm{IMC} \geq 30,0 \mathrm{Kg} / \mathrm{m}^{2}\right) \mathrm{para}$ adultos e, normal $\left(22,0 \mathrm{Kg} / \mathrm{m}^{2} \geq \mathrm{IMC}<27,0 \mathrm{Kg} / \mathrm{m}^{2}\right)$, sobrepeso $\left(27,0 \mathrm{Kg} / \mathrm{m}^{2} \geq \mathrm{IMC}<30,0 \mathrm{Kg} / \mathrm{m}^{2}\right)$ e obesidade $\left(\mathrm{IMC} \geq 30,0 \mathrm{Kg} / \mathrm{m}^{2}\right) \mathrm{para}$ idosos. ${ }^{* *}$ RCE: Normal (RCE $\left.<0,5\right)$, Aumentada $(\mathrm{RCE} \geq 0,5)$ para ambos os sexos. ${ }^{* * *} \mathrm{CC}$ : mulheres $<80 \mathrm{~cm}($ normal), $\geq 80 \mathrm{~cm} \mathrm{a}$ $<88 \mathrm{~cm}$ (aumentado) e $\geq 88 \mathrm{~cm}$ (muito aumentado) e, homens $<94 \mathrm{~cm}$ (normal), CC $\geq 94 \mathrm{~cm} \mathrm{a}<102 \mathrm{~cm}$ (aumentado) e $\geq 102 \mathrm{~cm}$ (muito aumentado). 
Tabela 2. Distribuição amostral e prevalência de risco nutricional combinado entre adultos residentes em Comunidades Quilombolas segundo diferentes critérios de combinação de risco e de acordo com características sociodemográficas. Estudo COMQUISTA, Bahia, 2011.

\begin{tabular}{|c|c|c|c|}
\hline \multirow[b]{2}{*}{ Variáveis } & \multirow{2}{*}{$\begin{array}{c}\text { Distribuição } \\
\text { amostral } \\
\text { n }(\%)\end{array}$} & \multicolumn{2}{|c|}{ Risco nutricional combinado } \\
\hline & & $\begin{array}{l}\text { IMC+RCE } \\
\%(\text { IC 95\%) }\end{array}$ & $\begin{array}{c}\text { IMC+CC } \\
\%(\text { IC 95\%) }\end{array}$ \\
\hline & & $\mathrm{n}=707$ & $\mathrm{n}=705$ \\
\hline Sexo & & $\mathrm{p}<0,001$ & $\mathrm{p}<0,001$ \\
\hline Masculino & $343(46,41)$ & $22,94(18,36-27,50)$ & $8,56(5,52-11,60)$ \\
\hline Feminino & $396(53,59)$ & $46,05(41,02-51,07)$ & $42,59(37,59-47,59)$ \\
\hline Faixa etária & & $\mathrm{p}=0,001$ & $\mathrm{p}=0,059$ \\
\hline $20-29$ & $144(19,49)$ & $22,46(15,46-29,46)$ & $16,67(10,41-22,91)$ \\
\hline $30-39$ & $164(22,19)$ & $41,67(33,89-49,44)$ & $28,85(21,70-35,99)$ \\
\hline $40-49$ & $150(20,30)$ & $41,55(33,40-49,69)$ & $30,28(22,68-37,87)$ \\
\hline $50-59$ & $104(14,07)$ & $42,72(33,10-52,33)$ & $29,13(20,29-37,95)$ \\
\hline$\geq 60$ & $177(23,95)$ & $30,36(23,37-37,34)$ & $28,92(21,98-35,84)$ \\
\hline Escolaridade & & $\mathrm{p}=0,056$ & $\mathrm{p}=0,158$ \\
\hline 0 & $271(36,92)$ & $31,37(25,65-37,08)$ & $26,38(20,93-31,81)$ \\
\hline 1 a 3 & $210(28,61)$ & $41,06(34,33-47,79)$ & $29,13(22,89-35,35)$ \\
\hline 4 a 7 & $185(25,20)$ & $37,71(30,50-44,92)$ & $28,57(21,84-35,29)$ \\
\hline$\geq 8$ & $68(9,26)$ & $26,15(15,36-36,93)$ & $15,38(6,52-24,23)$ \\
\hline Cor da pele & & $\mathrm{p}=0,878$ & $\mathrm{p}=0,895$ \\
\hline Preta & $287(39,37)$ & $36,00(30,30-41,69)$ & $22,74(22,41-33,05)$ \\
\hline Parda & $325(44,58)$ & $36,33(30,97-41,69)$ & $27,10(22,13-32,06)$ \\
\hline Branco & $97(13,31)$ & $32,26(22,68-41,82)$ & $23,66(14,95-32,35)$ \\
\hline Outras & $20(2,74)$ & $31,58(10,06-53,08)$ & $26,32(5,93-46,69)$ \\
\hline Renda & & $\mathrm{p}=0,186$ & $\mathrm{p}=0,763$ \\
\hline$\leq 50$ & $184(26,67)$ & $31,98(24,97-38,97)$ & $25,00(18,49-31,50)$ \\
\hline $51-119$ & $144(20,87)$ & $38,13(30,01-46,24)$ & $26,81(19,38-34,24)$ \\
\hline $120-268$ & $180(26,09)$ & $39,88(32,55-47,21)$ & $28,32(21,57-35,06)$ \\
\hline$\geq 269$ & $182(26,38)$ & $30,29(23,44-37,12)$ & $23,56(17,22-29,89)$ \\
\hline Índice de bens & & $\mathrm{p}=0,467$ & $\mathrm{p}=0,706$ \\
\hline$\leq 3$ & $236(31,94)$ & $31,96(25,76-38,16)$ & $25,23(19,44-31,01)$ \\
\hline 4 a 5 & $293(39,65)$ & $36,88(31,22-42,53)$ & $28,37(23,08-33,64)$ \\
\hline$\geq 6$ & $210(28,42)$ & $36,59(29,96-43,20)$ & $25,98(19,93-32,02)$ \\
\hline Estado Civil & & $\mathrm{P}<0,001$ & $\mathrm{p}<0,001$ \\
\hline Casado/Com união consensual & $473(64,01$ & $40,04(35,53-44,54)$ & $31,58(27,29-35,86)$ \\
\hline Solteiro/Separado/Divorciado/Viúvo & $266(35,99)$ & $26,80(21,28-32,31)$ & $18,07(13,25-28,88)$ \\
\hline Situação Ocupacional & & $p=0,001$ & $\mathrm{p}<0,001$ \\
\hline Trabalha & $365(49,39)$ & $29,61(24,86-34,35)$ & $16,48(12,62-20,33)$ \\
\hline Não trabalha & $374(50,61)$ & $41,26(36,07-46,44)$ & $37,46(32,35-42,57)$ \\
\hline
\end{tabular}

${ }^{\mathrm{s}} \mathrm{IMC}+\mathrm{RCE}$ : IMC $\left(\geq 25,0 \mathrm{Kg} / \mathrm{m}^{2}\right.$ para adultos $\mathrm{e} \geq 27,0 \mathrm{Kg} / \mathrm{m}^{2}$ para idosos $), \mathrm{RCE}(\geq 0,5)$. Nota: a soma do número total varia devido a perdas de informações. ${ }^{8}$ IMC+CC: IMC $\left(\geq 25,0 \mathrm{Kg} / \mathrm{m}^{2}\right.$ para adultos e $\geq 27,0 \mathrm{Kg} / \mathrm{m}^{2}$ para idosos), CC ( $\geq 80 \mathrm{~cm}$ para mulheres e $\geq 94 \mathrm{~cm}$ para homens). Nota: a soma do número total varia devido a perdas de informações.

neamente, mesmo reconhecendo que a combinação das medidas antropométricas pode trazer uma melhoria em termos de sensibilidade para identificar riscos agregados à saúde ${ }^{17,23,25}$. Na região Nordeste do país, 27,5\% (IC:23,0; 32,4) dos homens e $42,0 \%$ (IC:38,1; 45,9) das mulheres apresentaram excesso de peso e obesidade abdominal $^{25}$, o que corrobora com as diferenças de gênero encontradas no presente trabalho. Estudo desenvolvido com chineses mostrou que a com- binação da CC e IMC associou-se com um risco maior de hipertensão, diabetes e dislipidemia, quando comparado com a utilização unitária destas medidas ${ }^{34}$.

A utilização de dois indicadores nutricionais combinados, que diferem na utilização das variáveis que mensuram a obesidade abdominal, tal como utilizado neste estudo, torna-se de importante utilidade clínica para direcionar o critério de melhor aplicabilidade em identificar a preva- 
Tabela 3. Distribuição amostral e prevalência de risco nutricional combinado entre adultos residentes em Comunidades Quilombolas segundo diferentes critérios de combinação de risco e de acordo com características comportamentais e de saúde. Estudo COMQUISTA, Bahia, 2011.

\begin{tabular}{|c|c|c|c|}
\hline \multirow[b]{2}{*}{ Variáveis } & \multirow{2}{*}{$\begin{array}{c}\text { Distribuição } \\
\text { amostral } \\
\text { n }(\%)\end{array}$} & \multicolumn{2}{|c|}{ Risco nutricional combinado } \\
\hline & & $\begin{array}{l}\text { IMC+RCE } \\
\%(\text { IC 95\%) }\end{array}$ & $\begin{array}{c}\text { IMC+CC } \\
\%(\text { IC 95\%) }\end{array}$ \\
\hline & & $\mathrm{n}=707$ & $\mathrm{n}=705$ \\
\hline Tabagismo & & $\mathrm{p}=0,002$ & $\mathrm{p}<0,001$ \\
\hline Não & $585(79,16)$ & $38,31(34,25-42,36)$ & $30,09(26,26-33,91)$ \\
\hline Sim & $154(20,84)$ & $24,50(17,60-31,39)$ & $14,67(8,97-20,35)$ \\
\hline Atividade Física & & $\mathrm{p}=0,735$ & $\mathrm{p}=0,147$ \\
\hline Não realiza ou $\mathrm{faz}<3$ dias por semana & $569(78,70)$ & $35,72(31,68-39,76)$ & $28,10(24,29-31,89)$ \\
\hline$\geq 3$ dias/semana por $\geq 30 \mathrm{~min}$ & $154(21,30)$ & $34,22(26,57-41,88)$ & $22,14(15,44-28,49)$ \\
\hline Tempo de televisão & & $\mathrm{p}=0,207$ & $\mathrm{p}=0,002$ \\
\hline$<2 \mathrm{~h} /$ dia & $372(50,89)$ & $33,43(28,47-38,38)$ & $22,00(17,64-26,35)$ \\
\hline$\geq 2 \mathrm{~h} /$ dia & $359(49,11)$ & $38,00(38,89-43,10)$ & $32,18(27,25-37,10)$ \\
\hline Consumo de frutas & & $\mathrm{p}=0,253$ & $\mathrm{p}=0,493$ \\
\hline$<5$ dias/semanas & $603(81,93)$ & $36,32(32,39-40,23)$ & $26,25(22,65-29,84)$ \\
\hline$\geq 5$ dias/semanas & $133(18,07)$ & $30,89(22,68-39,10)$ & $29,27(21,18-37,35)$ \\
\hline Consumo de verdura/legumes & & $\mathrm{p}=0,733$ & $\mathrm{p}=0,756$ \\
\hline$<5$ dias/semanas & $636(86,41)$ & $35,13(31,33-38,92)$ & $26,72(26,20-30,24)$ \\
\hline$\geq 5$ dias/semanas & $100(13,59)$ & $36,96(27,02-46,89)$ & $28,26(18,99-37,52)$ \\
\hline $\begin{array}{l}\text { Comer frango com pele/carne vermelha } \\
\text { com gordura }\end{array}$ & & $\mathrm{p}=0,005$ & $\mathrm{p}=0,014$ \\
\hline Não & $417(58,57)$ & $31,59(27,03-36,15)$ & $23,50(19,33-27,66)$ \\
\hline Sim & $295(41,43)$ & $42,09(36,26-47,91)$ & $32,01(26,51-37,51)$ \\
\hline Auto-avaliação da saúde & & $\mathrm{p}=0,027$ & $\mathrm{p}=0,013$ \\
\hline Muito boa/Boa & $317(43,07)$ & $30,03(24,85-35,21)$ & $21,12(16,51-25,73)$ \\
\hline Regular & $319(43,34)$ & $40,39(34,88-45,89)$ & $31,37(26,15-36,58)$ \\
\hline Ruim/Muito Ruim & $100(13,59)$ & $36,17(26,38-45,95)$ & $30,11(20,71-39,49)$ \\
\hline Hipertensão Arterial & & $\mathrm{p}=0,013$ & $\mathrm{p}=0,002$ \\
\hline Não & $378(51,64)$ & $31,13(26,35-35,90)$ & $21,76(17,50-26,02)$ \\
\hline Sim & $354(48,36)$ & $40,12(34,88-45,35)$ & $32,34(27,33-37,35)$ \\
\hline Diabetes Mellitus & & $\mathrm{p}=0,550$ & $\mathrm{p}=0,151$ \\
\hline Não & $457(91,40)$ & $39,13(34,53-43,72)$ & $30,57(26,22-34,92)$ \\
\hline Sim & $43(8,60)$ & $43,90(28,48-59,32)$ & $41,46(25,15-56,76)$ \\
\hline
\end{tabular}

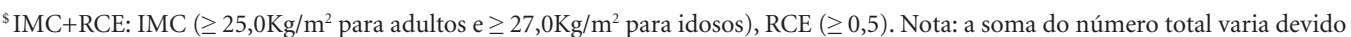
a perdas de informações. ${ }^{8}$ IMC+CC: IMC $\left(\geq 25,0 \mathrm{Kg} / \mathrm{m}^{2}\right.$ para adultos $\mathrm{e} \geq 27,0 \mathrm{Kg} / \mathrm{m}^{2}$ para idosos), CC ( $\geq 80 \mathrm{~cm}$ para mulheres $\mathrm{e}$ $\geq 94 \mathrm{~cm}$ para homens). Nota: a soma do número total varia devido a perdas de informações.

lência de excesso de peso e de obesidade central em homens e mulheres. Neste estudo, a medida que combina IMC + RCE foi a que conseguiu identificar melhor homens e mulheres com excesso de peso e obesidade abdominal concomitantemente. Uma das possíveis justificativas para esta situação pode ser atribuída ao fato da RCE ajustar o perímetro da cintura pela estatura, minimizando possíveis avaliações errôneas de riscos à saúde decorrentes da presença de indivíduos com diferentes estaturas ${ }^{35,36}$.

Ressalta-se que a simultaneidade da exposição ao excesso de peso e ao excesso de gordura abdominal, avaliada pelos dois tipos de indicadores nutricionais combinados investigados nesse estudo, aumentam o risco de DCNT e suas complicações. Os resultados mostraram alta prevalência de obesidade abdominal em mulheres e homens que tinham IMC normal. Estudo realizado com mulheres brasileiras em idade fértil mostrou que cerca de $1 / 4$ das mulheres sem excesso de peso apresentavam CC $>80 \mathrm{~cm}^{37}$. No Maranhão, foram encontrados 15,5\% de obesidade abdominal em mulheres eutróficas ${ }^{25}$. Estes resultados demonstram que o IMC isoladamente apresenta baixa sensibilidade para identificar o excesso de gordura corporal ${ }^{23,25,38}$, pois, apesar de considerar a proporção corporal, ele não informa adequadamente a presença de adiposidade e nem a distribuição da gordura corporal ${ }^{17}$, especial- 
Tabela 4. Fatores associados ao risco nutricional combinado segundo diferentes critérios de risco na análise multivariável entre adultos residentes em Comunidades Quilombolas. Estudo COMQUISTA, Bahia, 2011.

\begin{tabular}{|c|c|c|c|c|}
\hline \multirow[b]{3}{*}{ Variáveis } & \multicolumn{4}{|c|}{ Risco nutricional } \\
\hline & \multicolumn{2}{|c|}{$\mathrm{IMC}+\mathrm{RCE}^{\$}$} & \multicolumn{2}{|c|}{$\mathrm{IMC}+\mathrm{CC}^{\&}$} \\
\hline & $\begin{array}{l}\text { OR bruto } \\
\text { (IC95\%) }\end{array}$ & $\begin{array}{c}\text { OR ajustado* }^{*} \\
(\mathrm{IC} 95 \%)\end{array}$ & $\begin{array}{l}\text { OR bruto } \\
\text { (IC95\%) }\end{array}$ & $\begin{array}{l}\text { OR ajustado* } \\
\quad(\mathrm{IC} 95 \%)\end{array}$ \\
\hline \multicolumn{5}{|l|}{ Sexo } \\
\hline Masculino & 1,0 & 1,0 & 1,0 & 1,0 \\
\hline Feminino & $2,86(2,06-3,97)$ & $3,22(2,27-4,57)$ & $7,92(5,11-12,27)$ & $9,02(5,66-14,36)$ \\
\hline \multicolumn{5}{|l|}{ Faixa etária } \\
\hline $20-29$ & 1,0 & 1,0 & 1,0 & 1,0 \\
\hline $30-39$ & $2,46(1,47-4,10)$ & $2,65(1,50-4,68)$ & $2,02(1,15-3,57)$ & $2,19(1,13-4,23)$ \\
\hline $40-49$ & $2,45(1,45-4,13)$ & $3,07(1,68-5,52)$ & $2,17(1,22-3,85)$ & $3,30(1,69-6,72)$ \\
\hline $50-59$ & $2,57(1,47-4,50)$ & $3,18(1,63-6,21)$ & $2,05(1,10-3,80)$ & $2,81(1,28-6,17)$ \\
\hline$\geq 60$ & $1,50(0,89-2,52)$ & $1,93(0,98-3,77)$ & $2,03(1,16-3,55)$ & $3,02(1,38-6,64)$ \\
\hline \multicolumn{5}{|l|}{ Escolaridade } \\
\hline 0 & 1,0 & $1, .0$ & 1,0 & 1,0 \\
\hline 1 a 4 & $1,52(1,03-2,23)$ & $1,61(1,04-2,50)$ & $1,14(0,76-1,72)$ & $1,53(0,91-2,57)$ \\
\hline 4 a 7 & $1,33(0,88-1,98)$ & $1,76(1,07-2,89)$ & $1,11(0,72-1,71)$ & $1,79(1,00-3,19)$ \\
\hline$\geq 8$ & $0,77(0,41-1,43)$ & $1,94(0,91-4,12)$ & $0,50(2,44-1,05)$ & $1,59(0,62-4,05)$ \\
\hline \multicolumn{5}{|l|}{ Estado Civil } \\
\hline Casado/Com união consensual & 1,0 & 1,0 & 1,0 & 1,0 \\
\hline Solteiro/separado/divorciado/viúvo & $0,54(0,39-0,76)$ & $0,62(0,43-0,89)$ & $0,47(0,32-0,69)$ & $0,45(0,29-0,70)$ \\
\hline \multicolumn{5}{|l|}{ Tempo de televisão } \\
\hline$<2 \mathrm{~h} /$ dia & & & 1,0 & $1, .0$ \\
\hline$\geq 2 \mathrm{~h} / \mathrm{dia}$ & & & $1,68(1,19-2,36)$ & $1,67(1,12-2,48)$ \\
\hline \multicolumn{5}{|l|}{ Hipertensão Arterial } \\
\hline Não & 1,0 & 1,0 & 1,0 & 1,0 \\
\hline Sim & $1,48(1,08-2,02)$ & $1,57(1,08-2,30)$ & $1,71(1,22-2,40)$ & $1,70(1,10-2,64)$ \\
\hline
\end{tabular}

${ }^{5}$ IMC+RCE: IMC $\left(\geq 25,0 \mathrm{Kg} / \mathrm{m}^{2}\right.$ para adultos $\mathrm{e} \geq 27,0 \mathrm{Kg} / \mathrm{m}^{2}$ para idosos), RCE $(\geq 0,5) .{ }^{8} \mathrm{IMC}+\mathrm{CC}: \mathrm{IMC}\left(\geq 25,0 \mathrm{Kg} / \mathrm{m}^{2}\right.$ para adultos $\mathrm{e} \geq 27,0 \mathrm{Kg} / \mathrm{m}^{2}$ para idosos), $\mathrm{CC}$ ( $\geq 80 \mathrm{~cm}$ para mulheres $\mathrm{e} \geq 94 \mathrm{~cm}$ para homens). "OR ajustado: Odds Ratio ajustado por todas as variáveis incluídas no modelo.

mente no sexo masculino e em casos de sobrepeso e obesidade grau 1. Entretanto, sugere-se que o uso do IMC não deva ser negligenciado, pois, conjuntamente com indicadores de obesidade central contribuem para predizer gordura não abdominal, subcutânea, visceral e abdominal ${ }^{23,37}$.

As prevalências dos indicadores nutricionais combinados (IMC + RCE) e (IMC + CC) foram maiores entre as mulheres quilombolas. Em populações remanescentes de quilombos no Vale do Ribeira, em São Paulo ${ }^{39}$, as mulheres também apresentaram maiores prevalências de distúrbios nutricionais, possivelmente decorrentes da menor realização de atividade física no trabalho em comparação aos homens. Nestas comunidades, um grande percentual dos homens estava inserido em atividades agrícolas que requerem esforço físico mais intenso que o trabalho doméstico, que predomina nas mulheres quilombolas. Após a realização da análise da associação entre os indicadores nutricionais combinados com as variáveis de atividade física e ocupação estratificada pelo sexo (dados não mostrados), confirmou-se a assertiva acima de que nas comunidades quilombolas do Sudoeste da Bahia, as mulheres também apresentaram um maior percentual de inatividade física e um menor percentual de vinculação ao trabalho.

Contudo, a maior presença de excesso de peso entre mulheres não é achado universal no país. Em estudo de abrangência nacional, o excesso de peso foi mais freqüente em homens do que entre mulheres, excetuando-se as capitais de Salvador, São Paulo, Rio Branco e Recife ${ }^{40}$. A POF 2008$2009^{31}$ registrou maior prevalência de obesidade entre homens $(16,9 \%)$ do que entre mulheres $(12,5 \%)$, mas prevalência similar e próxima a $50 \%$ de sobrepeso nos dois sexos. Em contrapartida, em estudo conduzido nos seis municípios mais populosos do estado do Maranhão, a prevalência de obesidade abdominal pelo indicador CC em combinação com o excesso de peso pelo indicador IMC foi estatisticamente maior nas mulheres do que em homens ${ }^{25}$. 
Em relação à idade, o risco combinado IMC + RCE foi estatisticamente mais frequente na faixa de 40 a 59 anos, e o risco combinado IMC + CC, na faixa de 40 a 49 anos. O menor risco nos idosos não pode ser explicado por erros intrínsecos das medidas usadas. O impacto da perda de estatura decorrente do processo de envelhecimento ${ }^{41}$ sobre a prevalência de sobrepeso foi parcialmente considerado pelo ponto de corte do IMC, que foi mais elevado para os idosos (IMC $\geq 27 \mathrm{~kg} / \mathrm{m}^{2}$ ) do que para os adultos (IMC $\geq 25 \mathrm{~kg} / \mathrm{m}^{2}$ ). Entretanto, o mesmo não ocorreu com a medida RCE, que também é influenciada pela estatura. Portanto, é possível que a RCE aumentada esteja sobre-estimada em pessoas mais velhas, devido à redução do denominador da medida por perda na estatura com o avançar da idade. Por isto, o encontro de menores prevalências de distúrbio nutricional IMC + RCE nos mais velhos, mesmo com a possibilidade de erro, expressa diferenças reais de coorte de nascimento na exposição aos fatores que levam ao risco de obesidade, como o consumo de alimentos ultraprocessados e a inatividade física $^{42}$. Os indivíduos mais jovens possivelmente foram mais expostos aos modos de vida urbanos vigentes do que os mais velhos residentes nas comunidades quilombolas investigadas.

Maior chance de risco nutricional combinado IMC + RCE foi identificada entre indivíduos de maior escolaridade, contudo, na análise ajustada, a significância se deu apenas entre os indivíduos de menor escolaridade ( 1 a 7 anos de estudo), enquanto que para o risco IMC + CC maiores chances ocorreram na faixa de 4 a 7 anos de estudo, possivelmente devido ao pequeno número de pessoas com mais de 8 anos de estudo. Estudo prévio conduzido nacionalmente ${ }^{43}$ indica que determinados estratos sociais menos favorecidos da população brasileira, estão sofrendo intensificação da exposição aos fatores que levam à obesidade, como o sedentarismo e o baixo consumo de frutas e hortaliças, o que coaduna com os resultados encontrados nesta investigação.

Em relação ao estado civil, existe uma escassez de estudos que investigam a associação entre esta variável e indicadores antropométricos de sobrepeso/obesidade ${ }^{44}$ no Brasil. Gigante et $\mathrm{al}^{3}$, ao analisarem dados Vigitel 2006, identificaram que a prevalência de excesso de peso e obesidade foram mais elevadas entre homens e mulheres que referiram ter união estável, resultado que permaneceu mesmo após ajuste para a idade. No presente estudo, os quilombolas não casados também apresentaram menor chance de risco nutricional, mesmo considerando o efeito da idade. É possível que este resultado se deva a diferenças no modo de vida não captadas no presente estudo, como a alimentação e a atividade física.

Entre as variáveis comportamentais, apenas assistir televisão se manteve associada de forma independente ao risco nutricional combinado IMC + CC. Indivíduos que assistiam 2 horas ou mais de televisão por dia, fora do trabalho, tiveram chance 1,6 vezes de ter o risco nutricional combinado. Interessante destacar que a eletrificação no meio rural, ganhou impulso com o Programa Luz para Todos, o qual tem somente cerca de dez anos e a previsão para universalização da eletrificação rural ainda está prevista para 2014, incluindo comunidades quilombolas e outras comunidades de difícil acesso nas regiões Norte e Nordeste do país, as quais possuem maiores índices de população total em aéreas rurais ${ }^{45}$. Ademais, 85,4\% dos domicílios quilombolas investigados referiram possuir televisão, e cerca de 9,4\% referiram possuir duas ou mais unidades deste bem em seu domicílio. Nesta direção, as mulheres quilombolas foram as que mais horas permaneceram em frente da televisão fora do trabalho, quando comparadas com os homens (dados não mostrados), o que pode justificar, em parte, um maior percentual de obesidade entre elas. Assim, a importância para a saúde de assistir televisão fora do trabalho por um tempo prolongado, é que este tipo de atividade pode fomentar o sedentarismo e aumentar o consumo calórico, elevando o risco de obesidade ${ }^{46}$.

A associação entre hipertensão arterial e risco nutricional combinado em residentes em comunidades quilombolas foi confirmada nos dois modelos de regressão utilizados no presente estudo. A associação entre obesidade e hipertensão é bastante estabelecida e documentada no país ${ }^{3}$, o que justifica a integração desta morbidade ao plano de ações estratégicas das doenças crônicas não transmissíveis no Brasil ${ }^{47}$. Entre os quilombolas estudados, a prevalência de hipertensão foi considerada alta, alcançando a cifra de 45,4\% (IC95\%: 41,9-48,8). Fatores individuais (idade, classe econômica e escolaridade), fatores contextuais (segurança na vizinhança) e comportamentais (inatividade física e alimentação inadequada) foram apontados como determinantes da hipertensão arterial nesta população ${ }^{48}$.

\section{Conclusão}

Uma das possíveis limitações deste estudo inclui a ausência de aferição da confiabilidade das me- 
didas antropométricas, sendo esta falta minimizada pela padronização de procedimentos, assim como por medidas de garantia e de controle de qualidade, tornando possíveis erros de medida não diferenciais e as associações conservadoras. A dificuldade de se estabelecer relação temporal entre as variáveis de exposição e os desfechos analisados constituiu outra limitação na determinação de possíveis relações de causalidade. Além disso, taxas de não resposta mais elevadas entre homens e entre os mais jovens podem ter comprometido a aferição das diferenças de prevalências dos riscos nutricionais combinados (IMC + RCE) e (IMC + CC) entre os sexos.

$\mathrm{O}$ estudo mostrou elevada prevalência de risco nutricional para DCNT, em especial entre as mulheres residentes nas comunidades quilombolas estudadas. A utilização dos indicadores nutricionais combinados (IMC + CC) e (IMC + RCE), especialmente deste último, potencializa a detecção de indivíduos de alto risco para transtornos metabólicos e doenças associadas, uma vez que combina a gordura corporal e central. Considerando a precariedade de condiçoes de vida e saúde que caracterizam essas comunidades, recomenda-se que ações intersetoriais sejam deli- neadas e implementadas com vistas a promover estilos de vida saudáveis que contribuam para a redução do peso corporal e melhoria nutricional nas comunidades quilombolas, reduzindo assim as desigualdades sociais e de saúde no país.

\section{Colaboradores}

DA Soares trabalhou na concepção, delineamento, análise, interpretação dos dados, redação do artigo, aprovação da versão final do manuscrito. SM Barreto trabalhou na concepção, delineamento, análise, interpretação dos dados, revisão crítica e aprovação da versão final do manuscrito. 


\section{Referências}

1. Duncan BB, Chor D, Aquino EM, Bensenor IM, Mill JG, Schmidt MI, Lotufo PA, Vigo A, Barreto SA. Doenças crônicas não transmissíveis no Brasil: prioridade para enfrentamento e investigação. Rev Saude Publica 2012; 46(Supl.1):126-134.

2. Candib LM. Obesity and diabetes in vulnerable populations: reflection on proximal and distal causes. Ann Fam Med 2007; 5(6):547-556.

3. Gigante DP, Moura EC, Sardinha LMV. Prevalência de excesso de peso e obesidade e fatores associados, Brasil, 2006. Rev Saude Publica 2009; 43(Supl. 2):83-89.

4. Oliveira LPM, Assis AMO, Silva MCM, Santana MLP, Santos NS, Pinheiro SMC, Barreto ML, Souza CO. Fatores associados a sobrepeso e concentração de gordura abdominal em adultos na cidade de Salvador, Bahia Brasil. Cad Saude Publica 2009; 25(3):570-582.

5. Schneider HJ, Glaesmer H, Klotsche J, Bohler S, Lehnert H, Zeiher AM, Mars W, Pittrow D, Stalla GK, Wittchen HU, DETECT Study Gorup. Accuracy of antropometric indicators of obesity to predict cardiovascular risk J Clin Endocrinol Metab 2007; 92(2):589-594.

6. Schneider HJ, Friedrich N, Klotsche J, Pieper L, Nauck M, John U, Dorr M, Felix S, Lehnert H, Pittrow D, Silber S, Volzke H, Stalla GK, Wallaschofski H, Wittchen HU. The predictive value of different measures of obesity for incident cardiovascular events and mortality. J Clin Endocrinol Metab 2010; 95(4):1777-1785.

7. Boggs DA, Rosenberg L, Cozier Y, Wise LA, Coogan D, Ruiz-Narvaez R, Palmer JR. General and abdominal obesity and risk of death among Black women. $N$ Engl J Med 2011; 365(10):901-908.

8. Brasil. Decreto no 4.887, de 20 de novembro de 2003 . Regulamenta o procedimento para identificação, reconhecimento, delimitação, demarcação e titulação das terras ocupadas por remanescentes das comunidades dos quilombos de que trata o art. 68 do Ato das Disposições Constitucionais Transitórias. Diário Oficial da União 2003; 21 nov.

9. Leite IB. O projeto político quilombola: desafios, conquistas e impasses atuais. Rev Estudos Feministas 2008; 16(3):965-977.

10. Brasil. Secretaria Especial de Políticas de Promoção da Igualdade Racial. Relatório de ações realizadas. Ano base 2012. Brasília, 2013. [acessado 2013 jan 15]. Disponível em: http://www.seppir.gov.br/destaques/diagnostico- pbq -agosto2.pdf

11. Silva DO, Guerreiro AFH, Guerrero CH, Toledo LM. A rede de causalidade da insegurança alimentar e nutricional de comunidades quilombolas com a construção da rodovia BR-163, Pará, Brasil. Rev Nutr 2008; 21(Supl.0):83-87.

12. Instituto de Pesquisa Econômica Aplicada (Ipea). Quilombos das Américas: articulação de comunidades afrorrurais: documento sintese. Brasília: Ipea; 2012.

13. Guerrero AFH. Situação nutricional de populações remanescentes de quilombos do município de Santarém Pará, Brasil [tese]. Rio de Janeiro: Fundação Oswaldo Cruz; 2010

14. Salvo VLMA, Rodrigues D, Baruzzi RG, Gimeno SGA. Perfil metabólico e antropométrico dos Suyá. Parque indígena do Xingu, Brasil Central. Rev Bras Epidemiol 2009; 12(3):458-468.
15. Moura PG, Batista LRV, Moreira EAM. População indígena: uma reflexão sobre a influência da civilização urbana no estado nutricional e na saúde bucal. Rev Nutr 2010; 23(3):459-465.

16. Gimeno SGA, Rodrigues D, Pagliaro H, Cano EM, Lima EES, Baruzzi RG. Perfil metabólico e antropométrico de índios Aruák: Mehináku, Waurá e Yawalapití, Alto Xingu, Brasil Central, 2000 /2002. Cad Saude Publica 2007; 23(8):1946-1954.

17. Freitas SN, Caiffa WT, César CC, Faria VA, Nascimento RM, Coelho GLLM. Risco nutricional na população urbana de Ouro Preto, Sudeste do Brasil: Estudo de Corações de Ouro Preto. Arq Bras Cardiol 2007; 88(2):191-199.

18. Ashwell M. Obesity risk: important of the waist-to-height ratio. Nursing Standard 2009; 23(41):49-54.

19. Jassen I, Katzmarzyk PT, Ross R. Waist circumference and not body mass index explains obesity-related health risk. Am J Clin Nutr 2004; 79(3):379-384.

20. Rocha NP, Siqueira-Catania A, Barros CR, Pires MM, Folchetti LD, Ferreira SRG. Análise de diferentes medidas antropométricas na identificação de síndrome metabólica, com ou sem alteração do metabolismo glicídico. Arq Bras Endocrinol Metab 2010; 54(7):636-643.

21. Ashwell M, Gunn P, Gibson S. Waist-to-height ratio is a better screening tool than waist circumference and BMI for adult cardiometabolic risk factors: systematic review and meta-analysis. Obes Rev 2012; 13(3):275286.

22. Ashwell M, Hsieh SD. Six reasons why the waist-to-height ratio is a rapid and effective global indicator for health risks of obesity and how its use could simplify the international public health message on obesity. Int J Food Sci Nutr 2005; 56(5):303-307.

23. Zhu S, Heska S, Wang Z, Shen W, Alison DB, Ross R, Heymsfield SB. Combination of BMI and wais circumference for identifying cardiovascular risk factors in whites. Obes Res 2004; 12(4):633-645.

24. Arden CI, Katzmarzyk, Janssen I, Ross R. Discrimination of health risk by combined body mass index and waist circumference. Obes Res 2003; 11(1):135-142.

25. Veloso HJF, Silva AAM. Prevalência e fatores associados à obesidade abdominal e ao excesso de peso em adultos maranhenses. Rev Bras Epidemiol 2010; 13(3):400-412.

26. Bezerra VM, Medeiros DS, Gomes KO, Souzas R, Giatti L, Steffens AP, Kochergin CN, Souza CL, Moura CS, Soares DA, Santos LRCS, Cardoso LGV, Oliveira MV, Martins PC, Neves OSC, Guimarães MDC. Inquérito de Saúde em Comunidades Quilombolas de Vitória da Conquista/BA (Projeto COMQUISTA): aspectos metodológicos e análise descritiva. Cienc Saude Colet 2013; 19(6):1847-1847

27. Jelliffe DB. The assessment of nutritional status of the community. Geneva: WHO; 1966.

28. Sociedade Brasileira de Cardiologia; Sociedade Brasileira de Hipertensão; Sociedade Brasileira de Nefrologia. VI Diretrizes de Hipertensão Arterial. Arq Bras Cardiol 2010; 95(Supl. 1):1-51.

29. World Health Organization (WHO). Obesity: preventing and managing the global epidemic. Report of a WHO consultation on obesity. Geneva: WHO; 1998. 
30. Lipschitz DA. Screening for nutritional status in the elderly. Prim Care 1994; 21(1):55-67.

31. Instituto Brasileiro de Geografia e Estatística (IBGE). Pesquisa de orçamentos familiares- POF 2008/2009. Antropometria e estado nutricional de crianças, adolescentes e adultos no Brasil. Rio de Janeiro: IBGE; 2010.

32. Linhares RS, Horta BL, Gigante DP, Dias-da-Costa JS, Olinto MTA. Distribuição de obesidade geral e abdominal em adultos de uma cidade no Sul do Brasil. Cad Saude Publica 2012; 28(3):438-448.

33. Leite MS, Santos RV, Gugelmin AS, Coimbra Júnior CE. Crescimento físico e perfil nutricional da população indígena Xavante de Sangradouro-Volta Redonda, Mato Grosso, Brasil. Cad Saude Publica 2006; 22(2):265-227.

34. Hou X, Lu J, Weng J, Ji L, Shan Z, Liu J, Tian H, Ji Q, Zhu D, Ge J, Lin L, Chen L, Guo X, Zhao Z, Li Q, Zhou Z, Shan G, Yang Z, Yang W, Jia W; China National Diabetes and Metabolic Disorders Study Group. Impact of waist circumference and body mass index on risk of cardiometabolic disorder and cardiovascular disease in Chinese adults: a national diabetes and metabolic disorders survey. PloS ONE 2013; 8(3):e57319.

35. Souza TF, Nahas MV, Silva DAS, Peres MA. Fatores associadas à obesidade central em adultos de Florianópolis, Santa Catarina: estudo de base populacional. Rev Bras Epidemiol 2011; 14(2):296-309.

36. Al-Zufir BM, Aziz AA, Abdullah, Noor NM. Waist height ratio compared to body mass index and waist circumference in relation to glycemic control in Malay type 2 diabetes patients, Hospital Universiti Sains Malaysia. Int J Collaborative Res Int Med Public Health 2012; 4(4):406-413.

37. Meller FO, Ciochetto CR, Sandos LP, Duval PA, Duval PA, Vieira MFA, Schafer AA. Associação entre circunferência da cintura e índice de massa corporal de mulheres brasileiras: PNDS 2006. Cien Saude Colet 2014; 19(1):75-81.

38. Carrasco F, Reyes E, Rimler O, Rios F. Exactitud del índice de masa corporal en la predicción de la adiposidad medida por impedanciometría bioeléctrica. Arch Latinoam Nutr 2004; 54(3):280-286.

39. Volochko A, Batista LE. Saúde nos Quilombos. São Paulo: Instituto de Saúde, Secretária Estadual da Saúde de São Paulo; 2009. (Temas em Saúde Coletiva, 9).

40. Moura EC, Morais Neto OL, Malta DC, Moura L, Silva LN, Bernal R, Claro RM, Monteiro CA.Vigilância de fatores de risco para doenças crônicas por inquérito telefônico nas capitais do 26 estados brasileiros e no Distrito Federal (2006). Rev Bras Epidemiol 2008; 11(Supl. 1):20-37.
41. Siqueira VO, Costa BVL, Lopes ACS, Santos LC, LimaCosta MF, Caiaffa WT. Different equations for determining height among the elderly: the Bambuí cohort study of aging. Cad Saude Publica 2012; 28(1):125-134.

42. Wiest JM, organizador. Alimentos e alimentação enquanto saberes e fazeres quilombolas no Limoeiro do $\mathrm{Ba}$ cuparí. Porto Alegre: Evangraf; 2008.

43. Monteiro CA, Conde WL, Castro IRR. A tendência cambiante da relação entre escolaridade e risco de obesidade no Brasil (1975-1997). Cad Saude Publica 2003; 19(Supl. 1):67-75.

44. Velásquez-Meléndez JG, Pimenta AM, Kac G. Epidemiologia do sobrepeso e da obesidade e seus fatores determinantes em Belo Horizonte (MG), Brasil: estudo transversal de base populacional. Rev Panam Salud Publica 2004; 16(5):308-314.

45. Instituto Acende Brasil. A universalização dos serviços de distribuição de Energia Elétrica. Cadernos de Política Tarifária 2007; 2(1):373-387.

46. Poterico JA, Ortiz AB, Mola CL, Miranda JJ. Asociación entre ver television y obesidad en mujeres peruanas. Rev Saude Publica 2012; 46(4):610-616.

47. Brasil. Ministério da Saúde (MS). Plano de ações estratégias para enfrentamento das doenças crônicas não trasmissíveis (DCNT) no Brasil, 2011-2012. [2012 fev 2]. Disponível em: http://portal/saude/profissional/area. cfm?id_area $=818$

48. Bezerra VM, Andrade ACS, César CC, Caiffa WT. Comunidades Quilombolas de Vitória da Conquista, Bahia, Brasil: hipertensão arterial e fatores associados. Cad Saude Publica 2013; 29(9):1889-902.

Artigo apresentado em 09/03/2014

Aprovado em 03/08/2014

Versão final apresentada em 05/08/2014 